\title{
Drop Impact onto a Metallic Porous Layer: Effect of Liquid Viscosity and Air Entrapment
}

\author{
Cristina Boscariol ${ }^{1}$, Dipak Sarker ${ }^{2}$, Boseon Kang ${ }^{1,3}$, Cyril Crua ${ }^{1}$, Marco Marengo ${ }^{1}$
}

\author{
${ }^{1}$ School of Computing Engineering and Mathematics, University of Brighton, United Kingdom \\ ${ }^{2}$ School of Pharmacy and Biomolecular Science, University of Brighton, United Kingdom \\ ${ }^{3}$ Chonnam National University, Gwangju, Korea \\ ${ }^{*}$ Corresponding author: c.boscariol@brighton.ac.uk
}

\begin{abstract}
The drop impact onto porous surfaces has important applications in many fields, such as painting, paper coating, drug delivery and cosmetic sprays. In most of these applications, the optimisation of the deposition process is carried out empirically, without a proper understanding of the physics and a theoretical modelling of the spreading and the imbibition phenomena. The purpose of this study is to analyse droplet impacts on metallic meshes to define a general modelling strategy of the impact regimen on particular 2D regular porous surfaces. The application of this structure is relevant in process like filtration but also in the medical field, considering for example reconstructive surgery. By analysing the impact of droplets of water, acetone and a mixture of glycerol and water, having a diameter and an impact velocity in a range of $1.5-3 \mathrm{~mm}$ and $2-4 \mathrm{~m} / \mathrm{s}$, respectively, on meshes with a pore size ranging between 25 and $400 \mu \mathrm{m}$, a regime map was built considering 6 different impact outcomes. The outcomes were characterised by a deposition of the droplet on the substrate, or a partial imbibition, or a total imbibition. By increasing the impact velocity, a splash region was defined, which is still characterised by a final deposition, a partial imbibition and a total imbibition. It is found that the most influencing parameters are closely linked to the liquid properties and the impact velocity, more specifically liquid surface tension plays a major role in defining the impact outcome. In the case of Acetone, the lower surface tension brings to an almost instantaneous total imbibition whereas the experiments conducted using water and glycerol solution, showed a major distribution of the deposition regimes with respect to the other outcomes, due to the effect of a higher viscosity. It was found that the geometrical characteristics of the mesh such as pore size and wire diameter, play an important role as well in defining the total imbibition outcome. Finally, the defined transition maps, shows that for a certain combination of physical properties and initial condition, the outcome of the droplet impact is predictable.
\end{abstract}

\section{Keywords}

Drop impact, porous surface, metallic mesh, impact regimes, imbibition

\section{Introduction}

The phenomenon linked to droplet impact on porous surfaces has important applications in many fields. Considering the sheer number of practical applications that involve surfaces of this level of complexity, it is important to remark the fact that the number of parameters that can affect the impact outcome is vast. For this reason, a range of numerical and experimental investigations is still required, for example, to quantify the imbibition due to porosity and identify the outcome of the impacts [1] [6]. The understanding of the parameters that play the most important role in the evolution of the droplet inside the pore is still an open question. Depending on its size, the droplet spreading will be affected by porosity and its evolution might be modified by pore distribution. For example, in the distribution of agrochemicals, droplets are distributed as aqueous solution and sprayed on plants using pumps [2]. Focusing on environmental applications, it is possible to refer to the infiltration of rain and surface water into soil and the migration of oil in permeable porous media [3]. Porous surfaces find an application even in internal combustion engines. In fact, by using a layer with a specific porosity in cylinder-process, it is possible to obtain homogeneous and low emission combustion by enhancing fuel vaporization and distribution in space [4]. Another application is given by the deposition of dyes on papers in the ink-jet printing process [5]. In comparing the impact of droplets on rough and porous surfaces, Roisman et al. [6] developed a model describing the different regimes of splashing thresholds. In their model, it is shown that in the case of porous surfaces, a deposition outcome, without splash, is more probable considering the partial penetration of the liquid in the pore. They proposed an experimental map showing that two most significant parameters influencing the prompt splash-deposition are the Weber number and the ratio given by two geometrical characteristics linked to roughness. Neyval et al. [7], presented a numerical model, based on the finite volume method, to analyse the dynamics of the impact absorption of a liquid droplet impinging on a porous medium. To model the dynamics of the fluid flow, they enhanced the effects of surface 
tension and capillary forces. They compared their results with experimental data resulting in good agreement. Sahu et al. [8] analysed the impact of nanoparticle suspension into porous filter membranes focusing on penetration given by the hydrodynamic effect. This phenomenon is caused by the kinetic energy brought by a drop, which impacts on the porous media having a very small pore size with respect to drop size. They compared this aspect with the effects given by dynamic and capillary pressures and concluded that penetration into porous medium is possible when the dynamic pressure is higher than the capillary pressure, but also when hydrodynamic focusing, that occurs when the drop diameter is much larger than pore diameter, is observed. Kumar et al. [9] pointed out that the overall imbibition is influenced both by the material of the porous media and by capillary and showed that increasing drop size brought to slower imbibition. Karepetsas et al. [10] investigated droplet interaction, considering both smooth and structured surfaces. Specifically, they focused on the droplet sliding on an inclined surface. The model they performed, treats the liquid-gas and liquid-solid interfaces in a unified content and defines the dynamic contact angle by combining the action of disjoining and capillary pressure and viscous stresses without applying a boundary condition. They pointed out how dynamic hysteresis can be linked to the topography of the substrate and in the case of a structured surface, they predicted the effect of static hysteresis by observing that the droplet slides only beyond a certain critical inclination angle. Moquaddam et al. [11], focused on the regime of bouncing on macro-textured superhydrophobic surfaces that are characterised by a reduction of the contact time. They based the study of these effects using the entropic lattice Boltzman model for multiphase flows, investigating numerically a liquid droplet impacting a surface with tapered posts. They could focus on pancake bouncing phenomenon in complete detail. In this way, it was possible to accurately estimate the transformation of kinetic energy in surface tension and viceversa. This research aims at defining a general modelling strategy of the impact regimes given by experiments conducted on metal porous meshes, for different combinations of pore dimension, impact velocity, drop radius, liquid surface tension and viscosity.

\section{Material and methods}

The experimental analysis was conducted using three liquids: water, acetone and a solution composed by water and glycerol, to analyse the effect of viscosity and surface tension of liquid. The target surfaces were selected from a set of stainless steel metal meshes mainly used for filtration applications, with a range of pore size between 25 and $400 \mu \mathrm{m}$, purchased from (Plastock Plastok® Meshes and Filtration Ltd.). The characteristics of the meshes and liquid properties are listed in Table 1 and 2.

Table 1 Mesh characteristics.

\begin{tabular}{ccc}
\hline Sample Number & Pore Diameter $(\mu \mathrm{m})$ & Wire Diameter $(\mathrm{mm})$ \\
\hline 1 & 25 & 0.025 \\
2 & 50 & 0.036 \\
3 & 80 & 0.05 \\
4 & 100 & 0.065 \\
5 & 125 & 0.1 \\
6 & 150 & 0.1 \\
7 & 200 & 0.125 \\
8 & 250 & 0.1 \\
9 & 400 & 0.22 \\
\hline
\end{tabular}

Table 2 Liquid Properties

\begin{tabular}{cccc}
\hline Liquid & $\begin{array}{c}\text { Density } \\
\left(\mathrm{kg} / \mathrm{m}^{3}\right)\end{array}$ & $\begin{array}{c}\text { Viscosity } \\
(\mathrm{mPa} \mathrm{s})\end{array}$ & $\begin{array}{c}\text { Surface } \\
\text { Tension }(\mathrm{N} / \mathrm{m})\end{array}$ \\
\hline Water & 996 & 1 & 0.073 \\
Acetone & 793 & $0.30-0.543$ & 0.023 \\
Water \& Glycerol & 1118.6 & 10 & 0.067 \\
\hline
\end{tabular}

The experiments were organised in different groups. In order to obtain a range of impact velocity between $2 \mathrm{~m} / \mathrm{s}$ and $4 \mathrm{~m} / \mathrm{s}$, the height of release was varied between $20 \mathrm{~cm}$ and $80 \mathrm{~cm}$. Two different needle sizes were used to analyse the effect of drop diameter on the porous surface. 
The optical setup included a Photron Fastcam SA4 high speed camera (with a resolution of $1024 \times 800$ pixels), angled at $60^{\circ}$ with respect to the horizontal plane. The test area was illuminated using a custom-built high-speed LED light source, synchronised to the high-speed camera. The image analysis was conducted using a MATLAB code for image processing, capable to identify the dimension of the spreading, the droplet initial diameter, and the impact velocity by tracing its centre of mass. Table 4 and 5 report the error analysis for each measurement.

Table 4 Error Analysis of Impact Velocity for Water, Acetone and Water \& Glycerol

\begin{tabular}{ccccccc}
\hline $\begin{array}{c}\text { Height of } \\
\text { Release } \\
(\mathrm{cm})\end{array}$ & $\begin{array}{c}\text { Needle } \\
\text { Gauge No. }\end{array}$ & Liquid & $\begin{array}{c}\text { Mean } \\
\text { Velocity } \\
(\mathrm{m} / \mathrm{s})\end{array}$ & $\begin{array}{c}\text { Standard } \\
\text { Deviation } \\
(\mathrm{m} / \mathrm{s})\end{array}$ & $\begin{array}{c}\text { Maximum } \\
\text { Value }(\mathrm{m} / \mathrm{s})\end{array}$ & $\begin{array}{c}\text { Minimum } \\
\text { Value }(\mathrm{m} / \mathrm{s})\end{array}$ \\
\hline 20.3 & 21 & Water & 1.8 & 0.17 & 2.2 & 1.6 \\
44.3 & 21 & Water & 2.9 & 0.11 & 3.1 & 2.7 \\
80.3 & 21 & Water & 3.9 & 0.16 & 4.2 & 3.5 \\
20.3 & $26 \mathrm{~s}$ & Water & 1.9 & 0.11 & 2.1 & 1.7 \\
44.3 & $26 \mathrm{~s}$ & Water & 2.9 & 0.14 & 3.1 & 2.7 \\
80.3 & $26 \mathrm{~s}$ & Water & 3.9 & 0.16 & 4.6 & 3.6 \\
20.3 & 21 & Acetone & 1.9 & 1.9 & 2.1 & 1.7 \\
44.3 & 21 & Acetone & 2.9 & 2.9 & 3.2 & 2.7 \\
80.3 & 21 & Acetone & 3.9 & 3.9 & 4.1 & 3.7 \\
20.3 & $26 \mathrm{~s}$ & Acetone & 1.9 & 0.13 & 2.2 & 1.7 \\
44.3 & $26 \mathrm{~s}$ & Acetone & 2.9 & 0.17 & 3.2 & 2.7 \\
80.3 & $26 \mathrm{~s}$ & Acetone & 3.7 & 0.20 & 4.3 & 3.5 \\
20.3 & 21 & Water \& Glycerol & 1.8 & 0.07 & 2.1 & 1.7 \\
44.3 & 21 & Water \& Glycerol & 2.7 & 0.10 & 3.1 & 2.6 \\
80.3 & 21 & Water \& Glycerol & 3.7 & 0.07 & 3.9 & 3.5 \\
20.3 & $26 \mathrm{~s}$ & Water \& Glycerol & 1.8 & 0.12 & 2.0 & 1.6 \\
44.3 & $26 \mathrm{~s}$ & Water \& Glycerol & 2.7 & 0.15 & 3.1 & 2.5 \\
80.3 & $26 \mathrm{~s}$ & Water \& Glycerol & 3.8 & 0.11 & 3.9 & 3.6 \\
\hline
\end{tabular}

Table 5 Error Analysis of Initial Diameter for Water, Acetone and Water \& Glycerol

\begin{tabular}{cccccc}
\hline $\begin{array}{c}\text { Needle } \\
\text { Gauge No. }\end{array}$ & Liquid & $\begin{array}{c}\text { Mean } \\
\text { Diameter } \\
(\mathrm{mm})\end{array}$ & $\begin{array}{c}\text { Standard } \\
\text { Deviation } \\
(\mathrm{mm})\end{array}$ & $\begin{array}{c}\text { Maximum } \\
\text { Value }(\mathrm{mm})\end{array}$ & $\begin{array}{c}\text { Minimum } \\
\text { Value }(\mathrm{mm})\end{array}$ \\
\hline 21 & Water & 3.0 & 0.12 & 3.3 & 2.6 \\
$26 \mathrm{~s}$ & Water & 1.9 & 0.09 & 2.1 & 1.7 \\
21 & Acetone & 2.0 & 0.11 & 2.2 & 1.7 \\
$26 \mathrm{~s}$ & Acetone & 1.7 & 0.10 & 2.1 & 1.6 \\
21 & Water \& Glycerol & 2.9 & 0.06 & 3.1 & 2.8 \\
$26 \mathrm{~s}$ & Water \& Glycerol & 1.5 & 0.18 & 1.8 & 1.00 \\
\hline
\end{tabular}

\section{Results and discussion}

By observing the result of the experiments, it is possible to identify 6 different outcomes. For a lower velocity impact, these outcomes are a deposition, a partial imbibition and a total imbibition. For a higher velocity impact, it is possible to observe a transition to a splash region, which is still characterised by a final deposition, a partial imbibition and a total imbibition. The deposition outcome is characterised by the fact that, in a range of 15-30ms after the drop impacts on the substrate, and after the spreading and the recoiling, it is still possible to observe a liquid pancake on the surface without a proper imbibition, and the droplet recoils in an asymmetrical shape. On the opposite, for the imbibition, the liquid penetrates completely through the mesh pores after the impact. The partial imbibition can be considered as a transition outcome in which during the recoiling process after the impact, part of the liquid penetrates under the surface and part of the liquid is deposited on the substrate in a time range of 4-6ms. To distinguish the different outcome regimes, a first attempt was made, considering a dimensionless parameter given by the ratio between pore size and droplet size, $\boldsymbol{\beta}=\frac{\boldsymbol{D}_{p}}{\boldsymbol{d}}$. The results are shown in Figures 1 to 3 . 


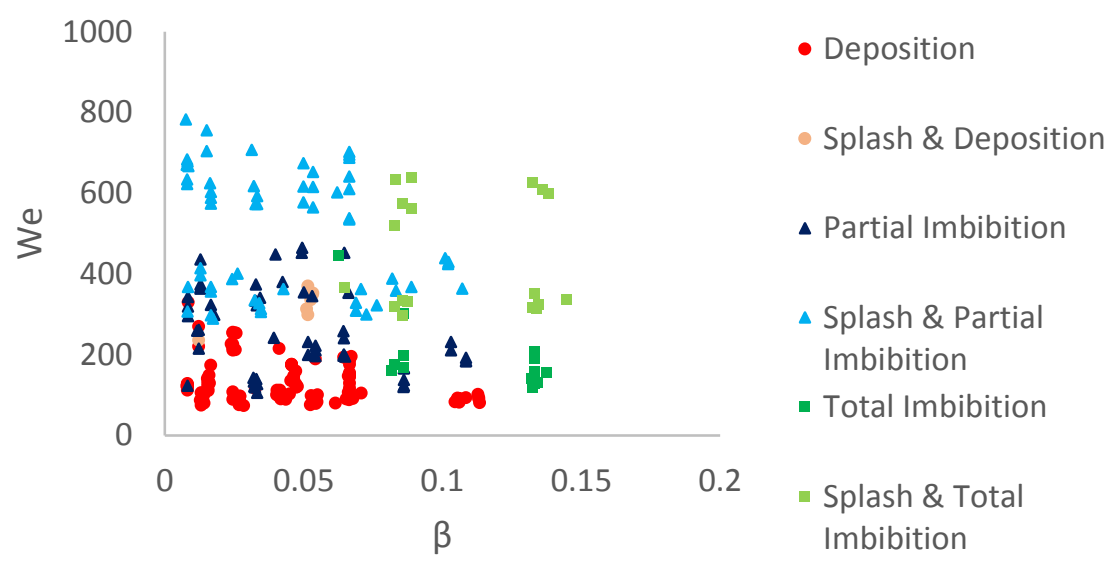

Figure 1 Regime distribution for water as a function of $\beta$.

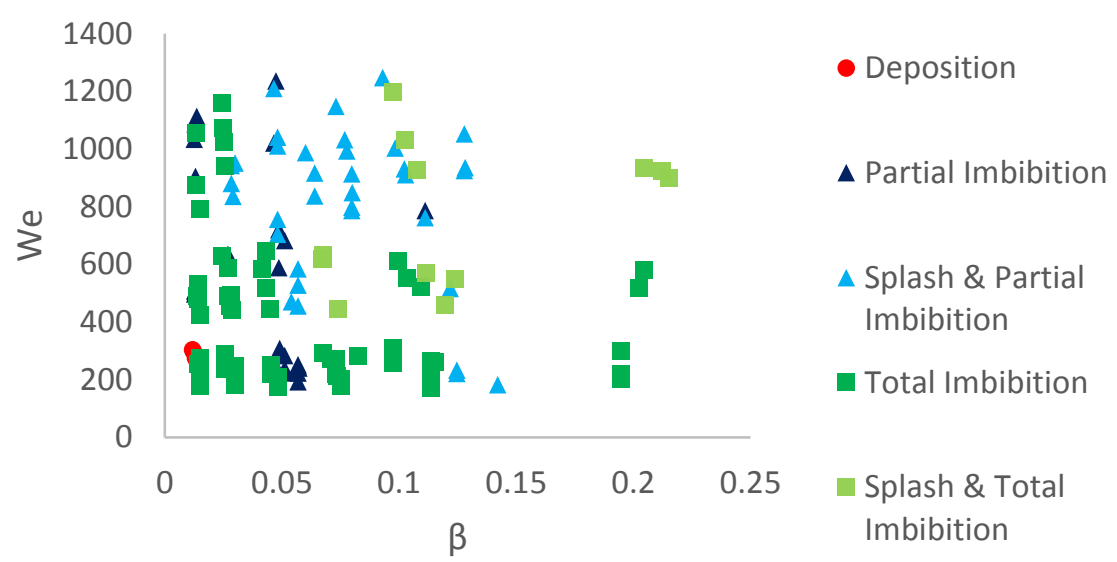

Figure 2 Regime distribution for acetone as a function of $\beta$

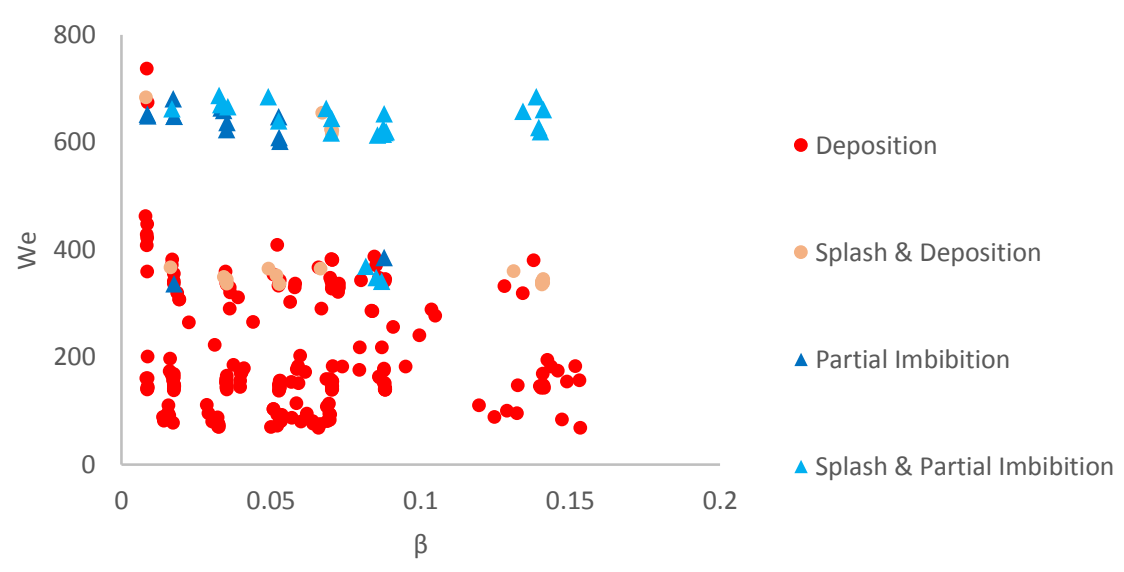

Figure 3 Regime distribution for water and glycerol as a function of $\beta$ 
From the figures above, it is possible to observe that in the case of water, an almost equal distribution of deposition, partial imbibition and total imbibition is achievable. On the contrary for the acetone, due to the lower surface tension of the liquid, the dominant outcome is given by a total imbibition. For the solution composed of water and glycerol, due to the higher viscosity of the liquid, the dominant outcome is given by the deposition. It can be seen that the separation of the outcomes is not clearly defined and, consequently, $\beta$ is not the best parameter to define the different outcome regions. A second attempt was made, introducing a new geometrical parameter given by the ratio of the empty area over the full area of the mesh pore, $\gamma=\left(1+2 \cdot \frac{D_{w}}{D_{p}}\right)^{2}$ where $D_{w}$ is the mesh wire diameter. This number was obtained considering that the ratio of the 2 areas can be written as $\frac{A_{\text {full }}}{A_{\text {empty }}}=\frac{\left(D_{p}+2 D_{w}\right)^{2}}{D_{P}^{2}}=D_{p}^{2} \cdot \frac{\left(1+\frac{2 D_{w}}{D_{p}}\right)^{2}}{D_{p}^{2}}=\left(1+\frac{2 D_{w}^{2}}{D_{p}^{2}}\right)$

Figure 4 shows schematically the interaction of the droplet with the mesh geometry.

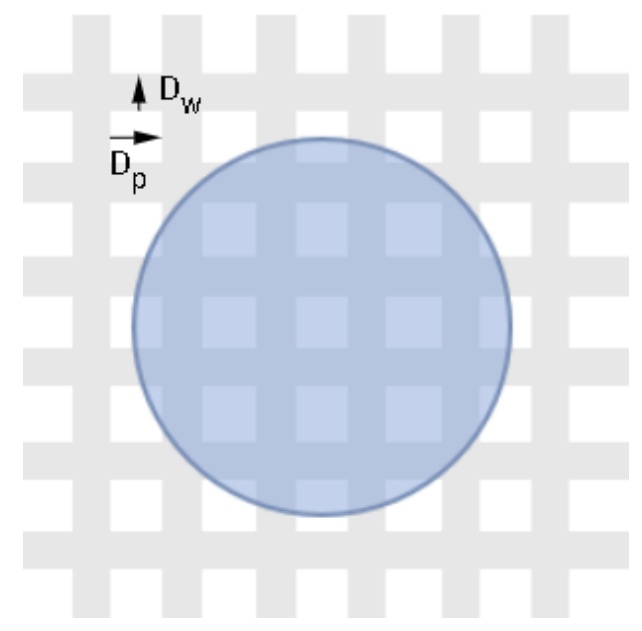

Figure 4 Impact and spreading of the droplet on the porous surface.

The results are shown in Figures 5.

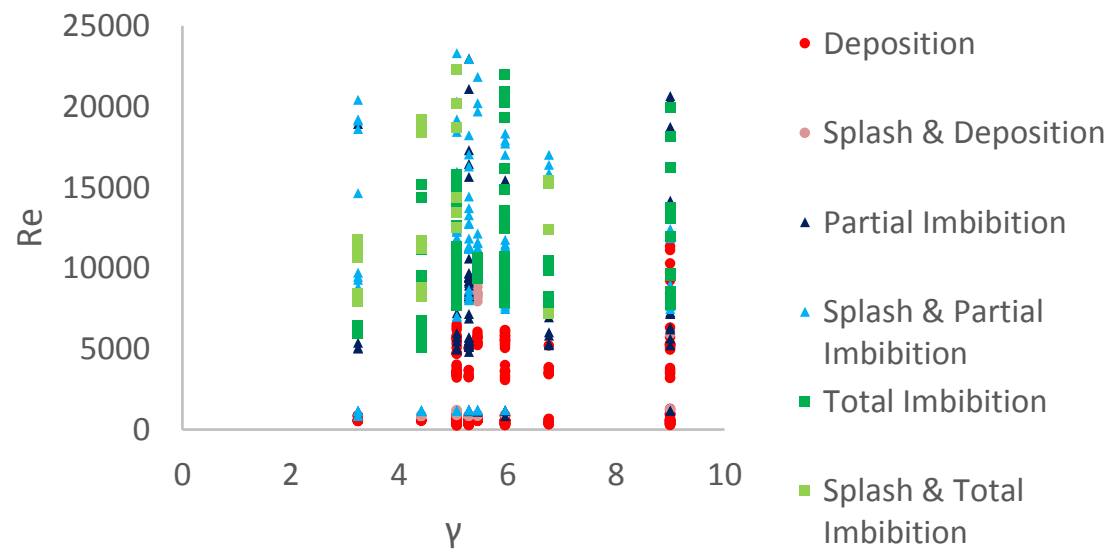

Figure 5 Regime distribution for all the liquids as a function of $\gamma$. It is shown that a clear distribution of the regions is still not achievable. 
By using $\gamma$, a clearer distribution of the outcomes is achieved in the case of water and a solution of water and glycerol but the case of acetone is still not clarified. Moreover, considering Figure 5, in which all the liquids data are reported, it is still not possible to clearly define the regions of deposition, partial imbibition and total imbibition having liquids with different characteristics of physical properties. Therefore, it is necessary to modify the dimensionless numbers used to define the regime map, and find a better combination capable to take in account of all the effects that can influence the impact outcome. Considering the previous results, it is possible to remark that $\gamma$, even if still not satisfactory to achieve the regime separations, plays an important role, under certain conditions, especially in the case in which the value of surface tension does not go under a certain value as for acetone. Consequently, it is possible to assume that the surface tension must be taken in consideration as well to define the regime distribution. Conversely, the splash region is mainly influenced by a higher impact velocity and a lower viscosity. It was chosen to represent the data introducing new dimensionless numbers. The dimensionless parameter on $\mathrm{x}$-axis is given by $\xi=p_{c}^{\star} \cdot \gamma$. Here, $p_{c}^{\star}$ is the capillary pressure $p_{c}=\frac{\sigma}{D_{p}}$ scaled by a critical value which still has to be empirically defined. In this way, the effect of geometry is coupled with the effects given by surface tension, in terms of imbibition effectiveness. The parameter on y-axis is given by $M=R e^{2} O h^{-1}$. In fact, considering the previous graph, it is clear that Reynolds number alone is not sufficient to obtain a satisfactory description of outcome distribution. For such reason it has been coupled with Ohnesorge number, which includes the surface tension effects. Even if this combination of dimensionless numbers brings to a satisfactory result (see Figure 6), it is still necessary to clarify which is the best value of the exponents attributed to Re and Oh to obtain the clearest separation of the different regimes.

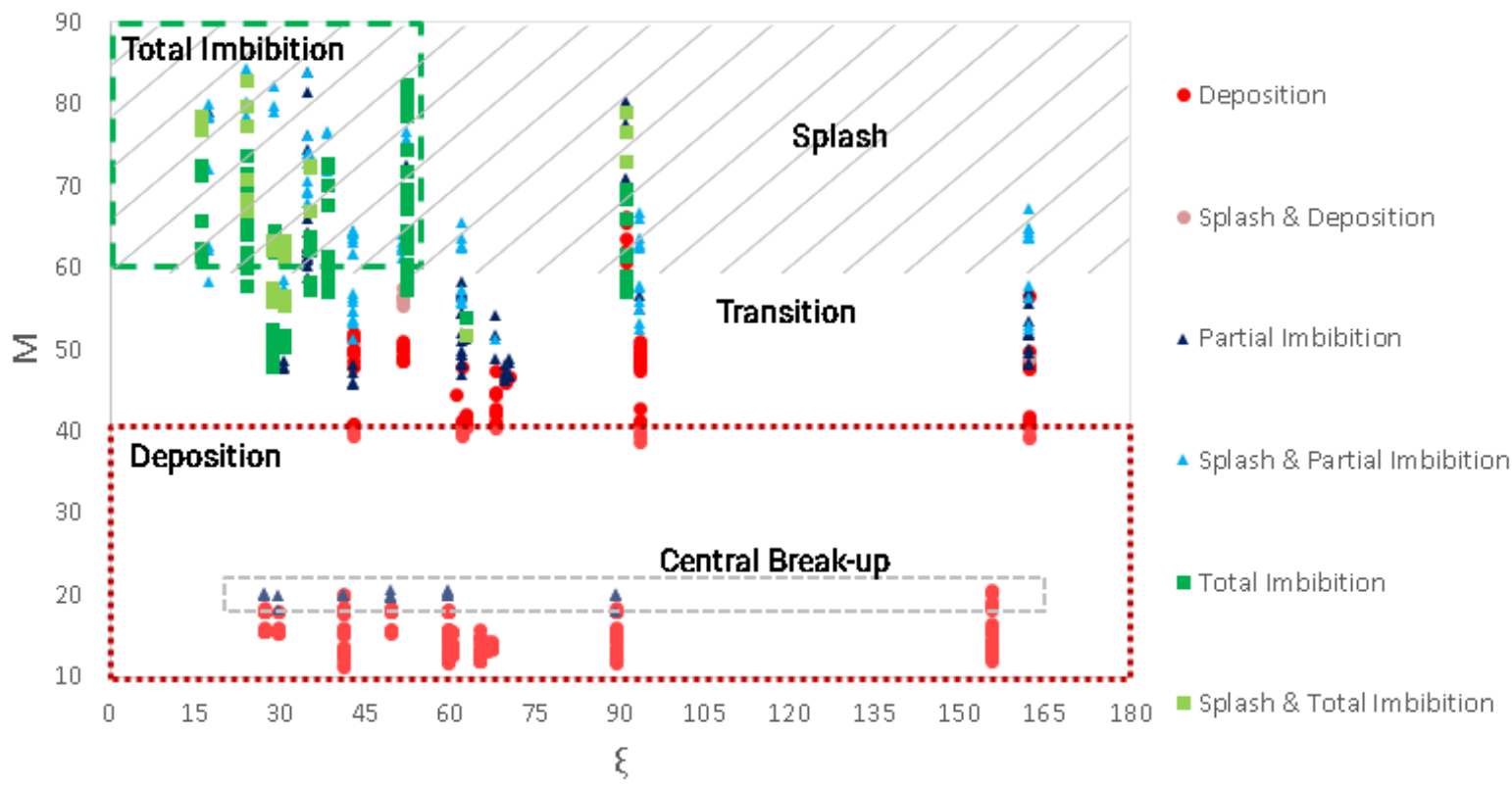

Figure 6 Regime distribution for all the liquids as a function of $\xi$ and $M$

It is now possible to observe that, the regime characterised by deposition, is localized in the lower part of the figure. In the middle part, a transition regime occurs, in which both deposition and partial imbibition are probable. In the upper part of the figure, a splash regime is identified, which is mainly characterised by a total imbibition for a value of $\xi<50$. In a small area in the deposition region, an outcome was detected characterised by a central break-up, mainly due to the experimental data collected for the solution composed by water and glycerol. Table 5 reports the region classification in terms of $\mathrm{M} / \xi$ space coordinates.

Table 5 Definition of regions in $M-\xi$ coordinates

\begin{tabular}{ccc}
\hline $\begin{array}{c}\text { Region } \\
\text { Definition }\end{array}$ & $M$ & $\xi$ \\
\hline Deposition & $0-45$ & $0-180$ \\
Transition & $45-55$ & $0-180$ \\
$\quad$ Total & $55-90$ & $0-50$ \\
Imbibition & $55-90$ & $0-180$ \\
Splash & 5
\end{tabular}




\section{Conclusions}

This study is focused on the investigation of droplet impact on metallic meshes with a wide range of pore sizes. It was found that the attempt to represent the different outcome regimes excluding a geometrical parameter referred to mesh wire diameter was not satisfactory to obtain a proper identification of regimes. In addition, to achieve a clear distinction of the impact regimes, it is fundamental to refer to a dimensionless number that also takes account the liquid properties, specifically, the viscosity and the surface tension. To reflect the above points, new dimensionless parameters, $\boldsymbol{M}$ and $\boldsymbol{\xi}$, are here introduced, and the outcomes of the impact are therefore predicted for the given range of experimental parameters. In considering this numbers, comparing the results to the literature, it was seen that conversely to what Roisman et al. pointed out in their study [6], our result show that the two most important parameters to describe the impact of droplet on a porous material may not be the We number and a ratio given by considering roughness geometrical parameter. The present results show a good agreement with Neyval et al. [7], and Sahu et al. [8], considering respectively the major role given to the surface tension, and the fact that an imbibition outcome is mainly observable for droplet with a larger diameter.

\begin{tabular}{ll}
\multicolumn{2}{l}{ Nomenclature } \\
$p_{c} \quad$ Capillary pressure $[\mathrm{Pa}]$ \\
$\sigma$ & Surface tension $[\mathrm{N} / \mathrm{m}]$ \\
$\rho$ & Liquid density $\left[\mathrm{kg} / \mathrm{m}^{3}\right]$ \\
$D_{p}$ & Pore diameter $[\mathrm{m}]$ \\
$D_{w}$ & Wire diameter $[\mathrm{m}]$ \\
$d$ & Droplet diameter $[\mathrm{m}]$ \\
$v_{i}$ & Impact velocity $[\mathrm{m} / \mathrm{s}]$ \\
$W e$ & Weber number \\
$\operatorname{Re}$ & Reynolds number \\
$\mu$ & Viscosity $[\mathrm{Pa} \mathrm{s}]$ \\
$\xi$ & $p_{c}^{\star} \cdot \gamma$ \\
$\mathrm{M}$ & $R e^{2} O h^{-1}$ \\
$\beta$ & $\frac{D_{\text {pore }}}{d}$ \\
$\gamma$ & $\left(1+2 \cdot \frac{D_{w}}{D_{\text {pore }}}\right)$
\end{tabular}

\section{References}

[1] Marengo, M., Antonini, C., Roisman, I. V., Tropea, C., (2011), Drop collision with simple and complex surfaces, Current Opinion in Colloid \& Interface Science, 16, pp. 292-302.

[2] Bertola, V., (2008), Some applications of controlled drop deposition on solid surfaces, Recent Patents on Mechanical Engineering, 1, pp. 167-174.

[3] Yarin, A.L., (2006), Drop impact dynamics: Splashing, Spreading, Receding, Bouncing, Annual Review of Fluid Mechanics, 38, pp. 159-192.

[4] Weclas, M., (2010), Potential of porous media combustion technology as applied to internal combustion engines, Journal of Thermodynamics, VOL 201039 pp.

[5] Woo Shik Kim, Sang Yong Lee, (2014), Behaviour of a drop impinging on heated porous surfaces, Experimental Thermal and Fluid Science, 55, pp. 72-70.

[6] Roisman, Ilia V., Lembach, A.Tropea, C., (2015). Drop splashing induced by target roughness and porosity: The size plays no role, Advances in Colloid and Interface Science ,VOL 222 p. 615-621.

[7] Neyval, C. R. Jr., Griffiths, R.F., Santos, J. M., (2004). Numerical simulation of the impact of liquid droplets on porous surfaces, Journal of Computational Physics,198, pp. 747-770.

[8]Sahu, R.P., Sett, S., Yarin, A.L., Pourdeyhimi, B., (2015), Impact of aqueous suspension drops onto nonwettable porous membranes: Hydrodynamic focusing and penetration of nanoparticles, Colloids and Surfaces A: Physicochem. Eng. Aspects, 467, pp. 31-45.

[9] Kumar, S., Deshpande, A.P.,(2006), Dynamic of drop srepading on fibrous porous media, Colloids and Surfaces A: Physicochem. Eng. Aspects, 277, pp. 157-163.

[10] Karapetsas, G., Chamakos, N. T., Papathanasiou, A.G., (2016),Efficient modelling of droplet dynamics on complex surfaces, Condens. Matter, 28, 16 pp.

[11] Mazloomi Moquaddam, A., Chikatamarla, Shyam S., Karlinm I., 2016, Drop bouncing off macro-textured superhydrophobic surfaces, Langmuir 